\title{
Surgery for Ovarian Cancer: Rationale and Guidelines
}

\author{
Benjamin E. Greer, MD, Ron E. Swensen, MD, and Heidi J. Gray, MD, Seattle, Washington
}

\author{
Key Words \\ Ovarian cancer, surgery, staging, cytoreduction, second-look \\ laparotomy
}

\section{Abstract}

Ovarian cancer is the second most common gynecologic cancer in women and the leading cause of death caused by gynecologic malignancy. Surgery plays a fundamental role in treating this challenging disease. Goals of primary surgery for ovarian cancer are to establish diagnosis, proper staging, determination of prognosis, and optimal cytoreduction of gross disease before chemotherapy for improved outcome. In addition to standard removal of the ovaries, uterus, omentum, and pelvic and para-aortic lymph nodes for early disease, extended surgical techniques used to debulk advanced disease include bowel resection, splenectomy, partial liver resection, peritoneal or diaphragmatic stripping, and use of laser or ultrasound (CUSA). Secondary surgery is used in a variety of situations. Second-look procedures were performed historically to determine response to chemotherapy to delineate duration of treatment, but now are best used in a research setting with the advent of improved chemotherapeutic agents. As a high percentage of patients have a gynecologic malignancy recurrence after primary treatment, many practitioners perform secondary cytoreductive procedures for recurrent disease. Additionally, in the recurrent setting, surgery may be necessary for relief of bowel obstruction and palliation of symptoms. Surgical management of ovarian cancer must be performed by surgeons, such as gynecologic oncologists, who have a firm understanding of the disease process, display good clinical judgment, and are adequately trained to perform the complex surgery that commonly is required for appropriate care. (JNCCN 2004;2:561-568)
\end{abstract}

From the Division of Gynecologic Oncology, Department of Obstetrics and Gynecology, University of Washington School of Medicine, Seattle, Washington.

Submitted August 6, 2004; accepted for publication September 15, 2004.

The authors have no financial interests, arrangement, or affiliations with the manufacturers of any products discussed in the article or with their competitors.

Correspondence: Benjamin E. Greer, MD, Professor, Division of Gynecologic Oncology, Department of Obstetrics and Gynecology, Box 356460, University of Washington School of Medicine, 1959 NE Pacific Street, Seattle, Washington 98195-6460. E-mail: bengreer@u.washington.edu bengreereu.washington.edu
Ovarian cancer accounts for the highest percentage of deaths from gynecologic cancers in the United States, with an estimated 26,738 women developing the disease in 2004 and 16,370 deaths. ${ }^{1}$ Surgery is an integral part of the management of ovarian cancer. Surgery establishes the diagnosis, provides appropriate staging, impacts outcome with optimal cytoreductive surgery, and, in appropriate clinical situations, is useful in the management of posttreatment evaluation and recurrent disease.

Ovarian cancer can be divided histologically into epithelial ovarian cancers, which affect approximately 90\% of women with this disease, and nonepithelial ovarian cancers. The nonepithelial ovarian cancers are sex cordstromal and germ cell tumors that usually occur in younger women, with different treatment strategies to maximize survival. The focus of this article is surgical principles for epithelial ovarian cancer, but typically these surgical principles can be applied to treating women with nonepithelial ovarian cancer as well as to women with ovarian sarcoma, borderline ovarian tumors, primary peritoneal cancer, and fallopian tube cancer.

\section{Surgical Staging: Early Ovarian Cancer}

Surgery establishes the correct stage of the ovarian cancer. This is important for prognosis and directing appropriate therapy. For example, well-differentiated intracystic ovarian cancer with negative staging biopsies (stage IA, grade I) has a high survival rate (around 95\%) and only requires observational follow up.

Understanding the early natural history and patterns of spread of epithelial ovarian cancer forms the basis for a rational system for surgical staging of apparent early ovarian cancer. Clinical observations made during the 1970s formed the basis of staging or restaging laparotomy for early ovarian cancer. ${ }^{2} \mathrm{~A}$ prospective trial of careful surgical staging procedures was performed 
Greer et al.

on patients believed to have stage I or II disease after initial surgery. In this series of 100 patients, 31\% of patients were upstaged as a result of additional surgery. Of these, $77 \%$ were reclassified as having stage III disease. ${ }^{3}$

This information was important in developing the 1987 staging system by FIGO (Federation Internationale de Gynecologie et d'Obstetrique) for ovarian cancer (stage IIIA was created by this information; Table 1). Histologic grade was an important predictor of occult metastasis: $16 \%$ of patients with grade 1 lesions, $34 \%$ with grade 2 , and $46 \%$ with grade 3 were upstaged. Before referral, only $25 \%$ of patients had initial surgical incision that was adequate for proper staging.

Since the FIGO report on surgical staging in early ovarian cancer, the importance of bilateral pelvic and para-aortic node dissection has been reported. In one study of 96 patients with gross disease confined to one ovary, and 15\% had microscopically positive lymph nodes. ${ }^{4}$ Fifty-four patients underwent bilateral node dissections; $19 \%$ had positive nodes and 30\% of these had contralateral disease. A recent report from the National Cancer Institute's SEER database indicated that only about $10 \%$ of American women with apparent early stage ovarian cancer received appropriate surgical staging and recommended postoperative therapy. ${ }^{5}$

Women with pelvic masses that may be ovarian cancer should undergo full evaluation and appropriate surgery by a qualified surgeon. Patients with elevated CA 125 or imaging studies suggestive of malignancy have indications for referral. Laparoscopic management of potentially malignant masses is not recommended.

Surgical laparotomy should be performed through a midline or para-median abdominal incision to allow adequate access to the upper abdomen. The ovarian tumor should be removed intact and a frozen section obtained. Patients with ovarian malignancy or at least borderline ovarian cancers and whose tumor is macroscopically confined to the ovaries or the pelvis should undergo meticulous surgical staging (Table 2).

\section{Table 1 FIGO Surgical Staging of Ovarian Carcinoma (1987)}

\section{Stage I}

Stage I ovarian cancer is limited to the ovaries.

IA: Tumor limited to one ovary; capsule intact, no tumor on ovarian surface. No malignant cells in ascites or peritoneal washings.

IB: Tumor limited to both ovaries; capsules intact, no tumor on ovarian surface. No malignant cells in ascites or peritoneal washings.

IC: Tumor limited to one or both ovaries with any of the following: capsule ruptured, tumor on ovarian surface, malignant cells in ascites or peritoneal washings.

Stage II

Stage II ovarian cancer is tumor involving one or both ovaries with pelvic extension and/or implants.

IIA: Extension and/or implants on the uterus and/or fallopian tubes. No malignant cells in ascites or peritoneal washings.

IIB: Extension to and/or implants on other pelvic tissues. No malignant cells in ascites or peritoneal washings.

IIC: Pelvic extension and/or implants (stage IIA or IIB) with malignant cells in ascites or peritoneal washings.

Stage III

Stage III ovarian cancer is tumor involving one or both ovaries with microscopically confirmed peritoneal implants outside the pelvis. Superficial liver metastasis equals stage III. Tumor is limited to the true pelvis but with histologically verified malignant extension to small bowel or omentum.

IIIA: Microscopic peritoneal metastasis beyond pelvis (no macroscopic tumor).

IIIB: Macroscopic peritoneal metastasis beyond pelvis $2 \mathrm{~cm}$ or less in greatest dimension.

IIIC: Peritoneal metastasis beyond pelvis more than $2 \mathrm{~cm}$ in greatest dimension and/or regional lymph node metastasis.

Stage IV

Stage IV ovarian cancer is tumor involving one or both ovaries with distant metastasis. If pleural effusion is present, there must be positive cytologic test results to designate a case to stage IV. Parenchymal liver metastasis equals stage IV.

Source: FIGO Cancer Committee, Gynecol Oncol 1986;25:383-385. 


\section{Table 2 Surgical Principals for Staging of} Apparent Early Ovarian Cancer

\section{Vertical incision}

Cytologic evaluation of ascites or washings

En-bloc and intact removal of tumor

Removal of remaining ovaries, uterus and tubes*

Examination of peritoneal surfaces with biopsy of adhesions or suspicious lesions

Infracolic omentectomy

Pelvic and para-aortic lymph node dissection

Random peritoneal biopsies of cul-de-sac, bladder peritoneum, paracolic gutters, and diaphragm

*May be preserved in selected patients.

At the time of initial opening of the peritoneum for a suspected ovarian malignancy, any free fluid or ascites should be collected for cytologic evaluation. If no free fluid is present, peritoneal washings should be obtained. After intact removal of the adnexa, if a cancer diagnosis is established, a total abdominal hysterectomy and contralateral salpingo-oophorectomy is performed. In select cases in which fertility preservation is desired, the uterus and opposite adnexa may be preserved. ${ }^{6}$ In particular, malignant germ cell neoplasms are unilateral and occur in young women who have not completed childbearing.

A systematic exploration of all intra-abdominal surfaces and viscera should be performed. This includes the peritoneal surfaces of the small and large bowel, including the mesentery, paracolic gutters, under surfaces of both hemidiaphragms, gall bladder, and liver. Any suspicious areas or adhesions should be biopsied. The peritoneum of the pelvic cul-de-sac, bladder peritoneum, and both paracolic gutters should be randomly biopsied. The diaphragm peritoneum should be biopsied or scraped for cytologic evaluation. An infracolic omentectomy should be performed. A pelvic and paraaortic lymph node dissection should be performed, unless there is a positive enlarged node on frozen section.

The final pathologic report of the comprehensive surgical staging provides accurate staging (Table 1). This provides the basis for most appropriate recommendation for adjuvant treatment. As indicated, patients with a stage IA grade 1 tumor can undergo observation. ${ }^{7}$ Other patients with stage I disease who are not on protocols are usually treated with three cycles of taxane and platinum agents. ${ }^{8}$ Because of a higher failure rate, stage II disease is managed similarly to more advanced ovarian cancers, with six to eight cycles of taxane and platinum-based chemotherapy.

Restaging surgical procedures should be performed in patients with apparent intracystic and grade 1 tumors that were not adequately staged with the initial surgery. Appendectomies should be performed in patients with mucinous tumors, because $8 \%$ of these patients will have involvement of the appendix. ${ }^{9}$ Routine appendectomy in early ovarian cancer is controversial and is performed in less than $4 \%$ of cases, ${ }^{10,11}$ although the appendix has been reported as the only site of extra-ovarian spread. ${ }^{12}$ Grade 3 tumors would be in indication for appendectomy.

\section{Advanced Ovarian Cancer: Cytoreductive Surgery}

Advanced ovarian cancer shows a varied spectrum of disease at surgical exploration. Maximal cytoreductive surgery has been the cornerstone of initial surgical management. The theoretical rationales for cytoreductive surgery are: (1) the physiologic benefit of removal of tumor mass or masses and ascites; (2) improved tumor perfusion with chemotherapy; and (3) improved immunologic competence of the patient. Cytoreductive surgery in advanced ovarian cancer is not adequate treatment. Chemotherapy should be started postoperatively. The current standard chemotherapy would be six cycles of paclitaxel and carboplatin..$^{13}$ The doses would be paclitaxel $175 \mathrm{mg} / \mathrm{m}^{2}$ over 3 hours and carboplatin AUC 5.0 to 7.5 every 3 weeks. Data exist that docetaxel $75 \mathrm{mg} / \mathrm{m}^{2}$ given over one hour could be substituted for paclitaxel ${ }^{14}$; prospective, randomized chemotherapy research trials integrating other active agents are ongoing. ${ }^{15}$

No prospective data are available on cytoreductive surgery, but retrospective evidence supports the advantages of such surgery. The goal of cytoreductive surgery is the removal of the primary cancer and all metastatic disease. This should include removal of the uterus and fallopian tubes as well as the ovaries. If complete resection of tumor is not technically feasible, the goal is to achieve optimal tumor reduction status. Definition of optimal tumor cytoreduction has varied over time. The concept was begun in the 1970s, when researchers noted that patients who underwent surgical cytoreduction of bulky disease to small-volume disease had a longer survival time. ${ }^{16}$ Traditionally, this 
Greer et al.

meant cytoreduction of all visible disease to a $2-\mathrm{cm}$ maximum diameter of individual aggregates of disease. ${ }^{17}$

Currently, 1-cm tumor diameter individual aggregates is considered optimal cytoreduction ${ }^{18}$; however, some reports support even smaller diameters. ${ }^{19,20}$ The number of individual aggregates present after a maximal cytoreductive surgery varies widely. For example, one patient may be considered optimally cytoreduced with only two residual tumor implants of 1 $\mathrm{cm}$ and another patient with 10 to 12 individual implants. Therefore, the distribution and extent of residual disease are important. ${ }^{21}$ In general, median survival doubles from 17 months to 39 months with aggressive surgery rendering the tumor down to optimal residuum. A meta-analysis of 6,885 patients found that maximal cytoreduction was one of the most powerful determinants of survival of patients with advanced ovarian cancer. Each 10\% increase in maximal cytoreduction was associated with a $5.5 \%$ increase in median survival time. The platinum dose intensity and cumulative dose had no effect on median survival time (that is, a cytoreduction of $75 \%=$ median survival of 37 months; cytoreduction of $25 \%=$ median survival of 23 months)..$^{22}$

The literature clearly shows that gynecologic oncologists are the most qualified and capable surgeons for attempting maximal cytoreduction surgery for ovarian cancer. To optimize the surgical results requires skills, experience, and judgment. The range of surgery for pelvic resection of uterus, tubes, and ovarian cancer may require modified or radical hysterectomy and even a posterior exenteration. In addition to omentectomy and pelvic and para-aortic node dissection, bowel resection or splenectomy may be indicated.

Ovarian cancer generally does not penetrate into the retroperitoneal space. Therefore, use of retroperitoneal surgical approach enables en bloc resection of pelvic disease. The ureters need to be identified and protected from injury. They are often distorted by tumor geometry and can be adherent to pelvic tumors.

The omentum in advanced ovarian cancer may be just studded with small metastasis or entirely replaced, forming an omental cake. One approach to removing the omentum is to dissect the omentum off the transverse colon in the avascular plane. Another approach is to open the lesser sac off the greater curvature of the stomach. If it is necessary to ligate the short gastric arteries, a nasogastric tube should be used postoperatively because of delayed gastric emptying.
The hilum of the spleen is often a site of extension of bulky omental ovarian metastasis. Splenectomy may be necessary to accomplish optimal cytoreduction or to control bleeding caused by traction on the splenic capsule. ${ }^{23}$ During splenectomy, care must be exercised to not damage the tail of the pancreas, which can lead to leak or fistulae. If the spleen is removed, immunization against Streptococcus pneumoniae, meningococcus, and Haemophilus influenzae should be performed postoperatively because these patients will be at higher risk for infection secondary to encapsulated organisms.

Retroperitoneal lymph nodes contain metastatic disease in up to $70 \%$ of patients with advanced ovarian cancer. In patents with isolated enlarged pelvic or para-aortic nodes, removal is mandatory in an optimal cytoreductive surgical effort. However, the role of systematic lymphadenectomy in patients with no palpably enlarged lymph nodes with advanced metastatic disease is unclear.

Advanced ovarian cancer often involves both the large and small bowel. The literature confirms that optimal cytoreduction to less than $1 \mathrm{~cm}$ of residual disease results in an improved survival of patients undergoing bowel resection at the time of primary surgery. ${ }^{24}$ Although ovarian cancer typically does not invade into the muscularis and mucosa of the intestine, clinically, metastatic implants that may exist in the bowel mesentery or serosa can cause obstruction or impending obstruction, and resection is indicated. In addition to the rectosigmoid, the most frequent sites of resection are the terminal ileum, cecum, and transverse colon. Resection of more than one segment may be necessary. Appendectomy should be performed if gross involvement with tumor is present or if the histology is a mucinous tumor.

Other ancillary procedures or techniques have been employed. These include diaphragm stripping and the use of argon beam coagulation or Cavitron ultrasonic surgical aspiration (CUSA). ${ }^{25-28}$ However, the efficacy of these procedures of improving outcome is unproven.

\section{Ovarian Cancer: Secondary Surgery}

Secondary surgery for epithelial ovarian cancer may be defined as surgery undertaken some time after the completion of primary staging surgery or cytoreductive surgery. This includes second-look laparotomy (SLL), 
secondary cytoreductive surgery, interval cytoreductive surgery, surgery for recurrent disease, and surgery for intestinal obstruction.

\section{Second Look Laparotomy}

SLL was incorporated into ovarian cancer management in the 1970s, when the appropriate duration of chemotherapy was unknown. At that time, the risks of iatrogenic malignancies were recognized with prolonged exposure to alkylating agents. ${ }^{29,30}$ Duration of treatment was directed by response as documented in a secondary surgical procedure. With the advent of platinum and paclitaxel-based primary therapy, concerns related to prolonged alkylating agent therapy have been overcome, and the role of SLL in directing treatment is diminishing.

The routine use of SLL in patients with ovarian carcinoma is controversial. Although clinicians may interpret the optimal purpose and timing of SLL with some variability, its definition as a comprehensive surgical staging procedure in a patient clinically free of disease (no evidence on physical examination, negative imaging studies, and normal tumor markers) is less ambiguous. As a noncurative procedure, its diagnostic value may be limited by random sampling techniques, and as a major surgical procedure, the potential exists for serious complications and impact on the patient's quality of life..$^{31}$ In addition, from a macroeconomic perspective, the costs associated with SLL are not inconsequential.

Despite these risks, SLL is the gold standard for identifying residual disease after standard first-line platinum-based chemotherapy. To benefit patients, SLL must be highly predictive of the presence or absence of disease and result in therapeutic decisions that improve survival.

In early stage disease, patients who have undergone appropriate ovarian cancer surgery and chemotherapy are not recommended for second-look surgery because of the low yield of positive findings indicating persistent disease. Two series reported in the literature regarding SLL in early stage ovarian cancers showed positive findings in only $5 \%$ and $12 \%$ of the surgeries, respectively. ${ }^{7,32}$

However, in advanced ovarian cancer, more than $50 \%$ of patients will have a positive second-look evaluation despite negative clinical evidence of disease. Additionally, the prognostic value is questionable because even among those with a negative SLL, the risk of recurrence is greater than $50 \%$. In reports of two
European multicenter randomized trials of SLL, neither study showed any survival benefit. ${ }^{33,34}$ These studies have been criticized because of methodologic flaws and the chemotherapy used.

Gynecologic Oncology Group (GOG) Protocol 158 was a randomized trial in patients with optimally resected stage III ovarian cancer whose primary objective was designed as a non-inferiority study to compare the efficacy and toxicity of carboplatin plus paclitaxel (arm II) with cisplatin plus paclitaxel (arm 1). Seven hundred ninety-two eligible patients were enrolled on the study. Prognostic factors were similar in the two treatment groups. Median PFS and overall survival was 19.4 and 48.7 months, respectively, for arm I compared with 20.7 and 57.4 months, respectively, for arm II. The relative risk (RR) of progression for the carboplatin plus paclitaxel group was 0.88 with a $95 \%$ confidence interval (CI) of 0.75 to 1.03 , and the RR of death was 0.84 with a $95 \% \mathrm{CI}$ of 0.70 to $1.02 . .^{13}$

SLL was not required but was used to assess response to treatment, which reflects the uncertainty of whether a second-look procedure enhances the overall treatment of patients with ovarian cancer. Patients were informed regarding the arguable value of second-look procedures before chemotherapy randomization. If they elected to undergo the procedure, they were registered with the GOG Statistical and Data Center (SDC). The choice was used as a stratification factor to insure balance of treatment assignment within the SLL and no SLL groups. In the absence of a randomized trial, GOG 158 provided a large prospective comparison of survival in patients who underwent SLL compared with those who were followed up clinically after completion of initial chemotherapy.

A nonrandomized, prospective comparison of progression-free survival (PFS) and survival in patients who underwent SLL versus patients who were followed up clinically was performed after completion of initial chemotherapy for optimally resected stage III ovarian cancer. Before chemotherapy randomization, patients participating in GOG Protocol 158 were informed of the controversies regarding SLL before choosing whether or not they would undergo the procedure (disease at SLL was not a study endpoint). Any chemotherapy initiated after finding disease at SLL or clinical recurrence was not standardized but physician-directed. The intent-to-treat approach 
customarily used in randomized clinical trials was replaced by explanatory analysis.

Three hundred ninety-three patients selected SLL and 399 selected no SLL. Median age of patients in the SLL group was 54 compared with 59 in the no SLL group, and patients selecting SLL were more likely than those in the no SLL group to have gross residual disease at initial surgery (69\% vs. 60\%, respectively), implying a need for adjusted analysis. The adjusted relative risk was .089 (95\% CI, 0.75-1.07) and the difference in median PFS was 1.0 month (SLL 23.9 vs. no SLL 22.9 months). No difference in survival was seen between the two groups. ${ }^{35}$

SLL combined with physician-directed secondline therapy for patients with positive SLL does not appear to substantially improve PFS or improve survival to any degree. SLL remains the gold standard for determining the presence or absence of disease. Ethically, for patient autonomy, SLL should be discussed because patients with a negative SLL result have a better prognosis. SLL can be used in patients with disease distribution that would make them candidates for consolidation treatment such as whole abdomen radiation. In summary, however, SLL has limited application with current chemotherapy agents and should be reserved for research purposes.

\section{Secondary and Interval Cytoreductive Surgery}

Interval cytoreductive surgery after neoadjuvant chemotherapy has been used in a number of clinical contexts. The most common clinical situation is for patients with suboptimal cytoreduction; after several cycles of chemotherapy, an attempt at further cytoreduction is made. The second most common context is for patients who are medically compromised and not candidates for initial radical cytoreduction because of risk of incurring severe morbidity. After six cycles of platinum-based chemotherapy, some practitioners will offer patients debulking surgery if they have had a good response with reduction in total bulk of disease. The third context is for patients who are electively treated with neoadjuvant chemotherapy and interval cytoreduction as primary therapy. These last two clinical situations are for patients who have had diagnosis by cytology or some type of biopsy.

Two large prospective randomized trials looking at outcomes in interval cytoreduction have been reported. An EORTC study of 319 patients showed a median survival time of 26 months for patients who underwent interval cytoreduction and 20 months for those who did not $(P=.04){ }^{36}$ This study involved patients with suboptimal initial surgery and three cycles of cisplatin and cyclophosphamide without progression before randomization. The GOG reported similar approach in 550 patients, but used three cycles of paclitaxel and cisplatin chemotherapy before surgery versus continued chemotherapy. The median survival time was 32 months for the interval group and 33 months for those receiving chemotherapy. ${ }^{37}$

Secondary cytoreduction surgery at the time of recurrence requires good judgment and experience. Patients who had complete or optimal resection at the primary surgery or a disease-free interval of more than a year are most likely to benefit. Patients should show clinical and radiologic evidence that the recurrent disease is resectable.

\section{Surgery for Intestinal Disease}

Intestinal obstruction is a common problem in ovarian cancer patients during the course of the disease. This clinical dilemma requires thoughtful evaluation and management before surgical intervention. Obstruction may be related to a mechanical blockage or to carcinomatosis. Initial management should be hydration and correction of any electrolyte abnormalities. Thorough imaging studies need to be performed to document type and number of obstructions and guide the decision to perform an exploratory procedure. Patients with very short life expectancy should not undergo exploration. If exploratory surgery is deemed appropriate, the range of procedures would include resection with anastomosis, colostomy, ileostomy, or jejunostomy. Gastrotomy tube placement may be indicated for palliation in some circumstances. Recently, intestinal stents have been placed using endoscopic procedures. Clinical experience, good judgment, and excellent surgical skills are critical to maximize outcome in this difficult clinical problem.

\section{Conclusions}

Surgery plays a vital role in the management of ovarian cancer. The goals include diagnosis, proper staging, therapeutic cytoreduction, and palliation. The rationale and guidelines have been outlined. To maximize outcomes for women with ovarian cancer, clinical experience, judgment, and a varied set of surgical skills are necessary. 
Surgery for Ovarian Cancer

\section{References}

1. American Cancer Society. Cancer Facts and Figures 2004. Washington, DC: The American Cancer Society; Available at: http://www.cancer.org. Accessed September 2004.

2. Greer BE, Rutledge FN, Gallager HS. Staging or restaging laparotomy in early-stage epithelial cancer of the ovary. Clin Obstet Gynecol 1980;23:293-303.

3. Young RC, Decker DG, Wharton JT, et al. Staging laparotomy in early ovarian cancer. JAMA 1983;250:3072-3076.

4. Cass I, Li AJ, Runowicz CD, et al. Pattern of lymph node metastases in clinically unilateral stage I invasive epithelial ovarian carcinomas. Gynecol Oncol 2001;80:56-61.

5. Munoz KA, Harlan LC, Trimble EL. Patterns of care for women with ovarian cancer in the United States. J Clin Oncol 1997;15:3408-3415.

6. Schilder J M, Thompson AM, DePriest PD, et al. Outcome of reproductive age women with stage IA or IC invasive epithelial ovarian cancer treated with fertility-sparing therapy. Gynecol Oncol 2002;87:1-7.

7. Walton L, Ellenberg SS, Major F Jr, et al. Results of secondlook laparotomy in patients with early-stage ovarian carcinoma. Obstet Gynecol 1987;70:770-773.

8. Bell J, Brady M, Lage J, et al. A randomized phase III trial of three versus six cycles of carboplatin and paclitaxel as adjuvant treatment in early stage ovarian epithelial carcinoma: A Gynecolog Oncology Group study (Abstr 1). Gynecol Oncol 2003;88:156.

9. Ayhan A, Tuncer ZS, Tuncer R, et al. Is routine appendectomy beneficial in the management of ovarian cancer? Eur J Obstet Gynecol Reprod Biol 1994;57:29-31.

10. Bese T, Kosebay D, Kaleli S, et al. Appendectomy in the surgical staging of ovarian carcinoma. Int J Gynaecol Obstet 1996;53:249-252.

11. Rose PG, Reale FR, Fisher A, et al. Appendectomy in primary and secondary staging operations for ovarian malignancy. Obstet Gynecol 1991;77:116-118.

12. Rose PG, Abdul-Karim FW. Isolated appendiceal metastasis in early ovarian carcinoma. J Surg Oncol 1997;64:246-247.

13. Ozols RF, Bundy BN, Greer BE, et al. Phase III trial of carboplatin and paclitaxel compared with cisplatin and paclitaxel in patients with optimally resected stage III ovarian cancer: A Gynecologic Oncology Group Study. J Clin Oncol 2003;21:3194-3200.

14. Vasey PA. Role of docetaxel in the treatment of newly diagnosed advanced ovarian cancer. J Clin Oncol 2003;21(suppl 10):S136-S144.

15. Bookman MA, Greer BE, Ozols RF. Optimal therapy of advanced ovarian cancer: carboplatin and paclitaxel vs. cisplatin and paclitaxel (GOG 158) and an update on GOG 182-ICON5. Int J Gynecol Cancer 2003;13:735-740.

16. Griffiths CT, Parker LM, Fuller AF Jr. Role of cytoreductive surgical treatment in the management of advanced ovarian cancer. Cancer Treat Rep 1979;63:235-240.

17. Smith JP, Day TG Jr. Review of ovarian cancer at the University of Texas Systems Cancer Center, M.D. Anderson
Hospital and Tumor Institute. Am J Obstet Gynecol 1979;135:984-993.

18. Omura GA, Brady MF, Homesley HD, et al. Long-term follow-up and prognostic factor analysis in advanced ovarian carcinoma: the Gynecologic Oncology Group experience. J Clin Oncol 1991;9:1138-1150.

19. Hacker NF, Berek JS, Lagasse LD, et al. Primary cytoreductive surgery for epithelial ovarian cancer. Obstet Gynecol 1983;61:413-420.

20. Hoskins WJ, Bundy BN, Thigpen JT, et al. The influence of cytoreductive surgery on recurrence-free interval and survival in small-volume stage III epithelial ovarian cancer: a Gynecologic Oncology Group study. Gynecol Oncol 1992;47: 159-166.

21. Farias-Eisner R, Teng F, Oliveira M, et al. The influence of tumor grade, distribution, and extent of carcinomatosis in minimal residual stage III epithelial ovarian cancer after optimal primary cytoreductive surgery. Gynecol Oncol 1994; 55:108-110.

22. Bristow RE, Tomacruz RS, Armstrong DK, et al. Survival effect of maximal cytoreductive surgery for advanced ovarian carcinoma during the platinum era: a meta-analysis. J Clin Oncol 2002;20:1248-1259.

23. Nicklin JL, Copeland LJ, O'Toole RV, et al. Splenectomy as part of cytoreductive surgery for ovarian carcinoma. Gynecol Oncol 1995;58:244-247.

24. Gillette-Cloven N, Burger RA, Monk BJ, et al. Bowel resection at the time of primary cytoreduction for epithelial ovarian cancer. J Am Coll Surg 2001;193:626-632.

25. Fiorica JV, Hoffman MS, LaPolla JP, et al. The management of diaphragmatic lesions in ovarian carcinoma. Obstet Gynecol 1989;74:927-929.

26. Brand E, Pearlman N. Electrosurgical debulking of ovarian cancer: a new technique using the argon beam coagulator. Gynecol Oncol 1990;39:115-118.

27. Deppe G, Malviya VK, Malone JM Jr. Use of Cavitron Ultrasonic Surgical Aspirator (CUSA) for palliative resection of recurrent gynecologic malignancies involving the vagina. Eur J Gynaecol Oncol 1989;10:1-2.

28. van Dam PA, Tjalma W, Weyler J, et al. Ultraradical debulking of epithelial ovarian cancer with the ultrasonic surgical aspirator: a prospective randomized trial. Am J Obstet Gynecol 1996;174:943-950.

29. Reimer RR, Hoover R, et al. Acute leukemia after alkylating-agent therapy of ovarian cancer. N Engl J Med 1977;297: 177-181.

30. $\overline{\text { Greene }} \mathrm{MH}$, Boice JD Jr, Greer BE, et al. Acute nonlymphocytic leukemia after therapy with alkylating agents for ovarian cancer: a study of five randomized clinical trials. N Engl J Med 1982;307:1416-1421.

31. Friedman JB, Weiss NS. Second thoughts about second-look laparotomy in advanced ovarian cancer. N Engl J Med 1990; 322:1079-1082.

32. Rubin SC, Jones WB, et al. Second-look laparotomy in stage I ovarian cancer following comprehensive surgical staging. Obstet Gynecol 1993;82:139-142. 
Greer et al.

33. Luesley D, Lawton F, et al. Failure of second-look laparotomy to influence survival in epithelial ovarian cancer. Lancet 1988;2(8611):599-603.

34. Fiorentino MV, Nicoletta MO, Tumolo S. Uselessness of surgical second-look in epithelial ovarian cancer: a randomized study (Abstr). Proc Am Soc Clin Oncol 1999;13:259.

35. Greer BE, Bundy BN, Ozols, RF, et al. Implications of secondlook laparotomy (SLL) in the context of Gynecologic Oncology Group (GOG) Protocol 158: a non-randomized comparison using an explanatory analysis (Abstr 2). Gynecol Oncol 2003;88:156-157.
36. van der Berg MEL, van Lent M, Buyse M, et al. The effect of debulking surgery after induction chemotherapy on the prognosis in advanced epithelial ovarian cancer. N Engl J Med 1995;332:629-634.

37. Rose $\mathrm{P}$, Nerenstone $\mathrm{S}$, Brady $\mathrm{M}$, et al. A phase III randomized study of interval secondary cytoreduction in patients with advanced stage ovarian carcinoma with suboptimal residual disease: a Gynecologic Oncology Group study (Abstr \#802). Program and abstracts of the American Society of Clinical Oncology, 38th Annual Meeting, May 18-21, Orlando FL, 2002. 